Cluster headache is uncommon in childhood. Onset is usually in the second and third decade. A retrospective review of cases attending a pediatric neurology clinic in Bristol, UK, between 2000 and 2005 identified 11 patients ( 7 male, 4 female) with median age of onset of 8.5 years (range 2-14). Median age at diagnosis was 11.5 years (range 7-17). Eight had episodic and 3 had chronic cluster headache. Most had cranial autonomic activation and agitated movement. (Majumdar A, Ahmed MA, Benton S. Eur J Paediatr Neurol 2008; Dec 22. (Epub ahead of print).

Maytal $\mathrm{J}$ et al modifications of the IHS criteria for pediatric migraine found that decreasing the length of attacks below 2 to 48 hours would increase the sensitivity of diagnosis, but adding associated autonomic symptoms of facial redness or pallor, while improving sensitivity, also decreased the specificity. The addition of CAS while helpful was not recommended. (Neurology 1997;48:602-607). Perhaps more attention to autonomic symptoms and behavior in diagnosis of children with migraine would be warranted.

\title{
TOPIRAMATE-INDUCED COUGH IN MIGRAINE PROPHYLAXIS
}

Three adults who developed intractable cough during topiramate prophylaxis of migraine are reported from the University of Padua and other centers in Italy. Cough developed early during the titration phase at dose levels of 75-100 mg/day, and resolved rapidly after withdrawal. Secondary causes of cough, including GERD, were excluded. The cough was episodic, dry, and very annoying, especially at night. Despite effective prevention of headache with topiramate, treatment was discontinued. Literature review revealed no previous case reports of cough as a side effect of topiramate treatment for migraine. (Maggioni F, Mampreso E, Mainardi F, Lisotto C, Malvindi ML, Zanchin G. Topiramateinduced intractable cough during migraine prophylaxis. Headache Oct 2009; on line). (Respond: Dr F Maggioni, Dept Neurosciences, University of Padua, Via Giustiniani 5, Padova, 35128, Italy).

COMMENT. Topiramate is a first-line treatment for migraine prophylaxis in adults. Adverse events in 20-25\% of patients may require discontinuation of treatment but are rarely severe. They include weight loss, dizziness, somnolence, paresthesias, impaired concentration and memory, and language difficulties. Cough has not been reported and the mechanism is unexplained. No patient received ACE inhibitors for hypertension, a known cause of dry cough in adults. Pubmed search for cough with topiramate treatment of childhood epilepsy or migraine found no reports.

\section{NEUROBEHAVIORAL DISORDERS}

\section{ISOLATED EPILEPTIFORM EEG DISCHARGES AND AUTISM}

The relationship between EEG abnormalities and neuropsychiatric disorders, and their possible clinical significance are reviewed by an investigator at Wayne State University, Detroit, MI, with special attention to the EEG and autism. Approximately one third of children with autistic spectrum disorder (ASD) develop epilepsy. Of 46 consecutive children

with autism (34 boys, and 12 girls, mean age $7.8+/-2.7$ years), 35\% had epilepsy (Canitano 